\title{
Performance Analysis of the Bluetooth Physical Layer
}

\author{
Aditya Karnik and Anurag Kumar \\ Dept. of Electrical Communication Engg. \\ Indian Institute of Science, Bangalore, 560012, INDIA \\ email: \{karnik, anurag\}eece.iisc.ernet.in
}

\begin{abstract}
Bluetooth is an emerging, short range, indoor wireless network technology, based on FH-CDMATTDD, which allows a collection of devices to form small overlapping networks, called piconets, in an ad hoc fashion. It is likely that the Bluetooth specification may be adopted in the IEEE 802.15 Wireless Personal Area Network standard. In this paper, we present an analysis of the Bluetooth physical layer. We consider a single room office environment (LOS propagation), with a number of piconets distributed in it. In the present work we have focussed only on the interference between piconets. Assuming a uniform distribution of Bluetooth devices in the room, we provide an analysis of the outage probability as a function of the number of overlapping piconets. We then characterise the temporal correlation in the outage process. This leads to an approximation for the méan outage duration. Combining the outage probability with the mean outage duration, we provide a 2-state Markov model for the Bluetooth channel.
\end{abstract}

\section{INTRODUCTION}

Bluetooth is an emerging indoor wireless network technology which aims at replacing many proprietary cables that connect one device to another with one universal short-range radio link. Various devices such as laptops, cell-phones, PDAs and desktops can be part of a Bluetooth system. Beyond untethering devices, Bluetooth also provides a mechanism to form small, ad-hoc networks of devices called piconets. Because of the ad-hoc nature of their formation, piconets are arbitrarily distributed in space and in fact form a system of overlapping networks. Moreover, a piconet is an autonomous network with a central controller and operates using FH-CDMA. One research objective is to characterise the behaviour of the wireless channel associated with piconets in order to understand the performance of applications over Bluetooth.

In this paper we study the performance of the hopping channel of a piconet as the number of piconets increases in a given area. We consider a typical scenario for Bluetooth, namely an office environment with piconets randomly distributed in it. The performance measure we use is the outage probability with respect to a target signal-to-noise ratio at the receiver. We find that the outage probability is of the order of $10^{-2}$ and expect it to increase linearly with the number of piconets. We then characterise the temporal correlation in the outage process. This leads to an approximation for the mean outage duration. Combining the outage probability with the outage du-

A part of this work was done during this author's summer internship in IBM, India Research Lab, New Delhi. ration, we provide a 2-state Markov model for the Bluetooth channel. Markov models for wireless channels ([1], [2]) are of interest because of their analytical simplicity. Such models would be useful, for example, in the performance analysis of TCP controlled applications over Bluetooth (see [3]).

The paper is organised as follows. In Section II, we briefly describe the Bluetooth physical layer. In Section III, indoor propagation models are discussed. In Section IV, we analyse the statistics of the outage process and provide a 2-state Markov channel model based on it. Results are provided in Section V, and we conclude in Section VI.

\section{BLuetooth Physical LAYER}

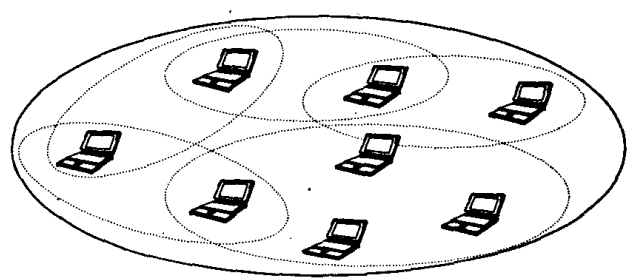

Fig. 1. In a Bluetooth network piconets overlap to form a scatternet.

Bluetooth ([4]) is a short range $(<10 \mathrm{~m})$ radio technology which operates in the $2.4 \mathrm{GHz}$ ISM band. Since the ISM band is free for use, there are many sources of interference, e.g., microwave ovens, WLAN etc. In addition, Bluetooth uses low power; in the range of $1 \mathrm{~mW}-100 \mathrm{~mW}$. To avoid interference, the physical layer is based on FH-CDMA. Bluetooth uses 79 carrier frequencies and as per the regulations, the signal bandwidth is limited to $1 \mathrm{MHz}$. Nominal bit rate is $1 \mathrm{Mbps}$. Sensitivity level (the input level for which the raw BER specification is met) and co-channel interference resistance are specified as $-70 \mathrm{dBm}$ and $11-14 \mathrm{~dB}$ respectively for $\mathrm{BER}<0.001$.

Figure 1 shows a network of overlapping Bluetooth piconets. By definition, the unit that establishes and coordinates a piconet is called the master. All other participants in the piconet are called slaves. There can be a maximum of 7 slaves in a piconet. A slow frequency hopping channel is associated with every piconet; each piconet has a unique hopping sequence determined by the master. Time is divided into slots of $625 \mu \mathrm{s}$. The timing is maintained by the master and slaves synchronise to the clock of the master.

Higher layer packets are fragmented into Bluetooth physical layer packets. Each packet has 126 bits of control informa- 
tion and a maximum payload of 2745 bits. Packet transmission takes place on one hop frequency and can take multiple slot. Full duplex communication is achieved by implementing TDD. Access control is exercised by the master. Only communication between the master and a slave is possible. The master transmits in odd numbered slot and the corresponding slave responds in the next slot. $1 / 3$ or $2 / 3$ FEC can be used for data. In addition, 1-bit acknowledgment indication ARQN is implemented. If the data is received incorrectly, it is retransmitted at the next opportunity.

\section{Modelling Indoor Wireless PRopagation}

Propagation of radio waves inside a building is a very complicated process, and it depends significantly on the indoor environment (e.g. office, factory), and topography (LOS, obstructed). The statistics of the indoor channel varies with time due to motion of people and equipment. A survey of indoor propagation measurement and models is provided in [5].

The indoor channel is characterised by high path losses and large variations in losses. The reported values of the path loss exponent $\eta$ are 1.5-1.8 for LOS and 2.4-2.8 for obstructed factory channels. In a factory environment ([6]), the overall path loss is found to be log-normal with $7.1 \mathrm{~dB}$ standard deviation, whereas in an office environment ([7]), the signal envelope is found to be Rician distributed ( $K$ ratio of about $2 \mathrm{~dB}$ ) around the mean determined by the propagation losses.

Motion within the building causes signal fading which compares well with the Rician distribution with $K$ equal to $6.8 \mathrm{~dB}$ and $10 \mathrm{~dB}$ for office and factory environments respectively ([6], [7]). If the degree of motion is small (as in an office environment), the fading is extremely slow; the Doppler bandwidth is about $4 \mathrm{~Hz}$. The channel is then said to be quasistatic. The fading statistics are, however, statistically nonstationary. but wide-sense stationary characteristics are exhibited for periods up to $2 \mathrm{~s}$. Since many digital symbols are transmitted during this period at practical data rates, this type of fading is referred to as quasi-wide-sense stationary.

In our analysis, since we consider LOS propagation in an open office environment, we take $\eta=2$ and a Rician signal distribution with $K=6 \mathrm{~dB}$.

\section{SIR ANALYSIS OF OVERLAPPING FH-CDMA PICONETS}

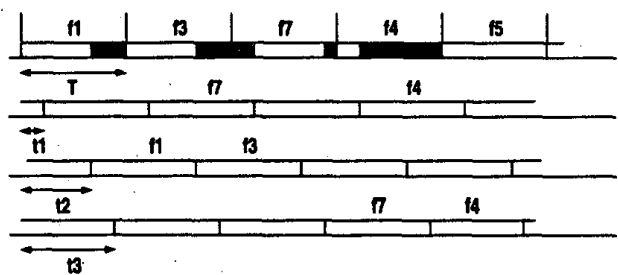

Fig. 2. Frequency hopping system. Black boxes indicate overlap of hop frequencies.
We consider a single circular room (radius $R$ ) office environment (LOS propagation), with a number of Bluetooth devices distributed randomly in it to form $M$ piconets. Because of the ad-hoc nature of the formation of piconets, devices in a piconet are spatially randomly distributed in the room leading to overlap among the piconets. Note that, in our results $M$ will be a parameter, i.e. we are interested in analysing the performance of the Bluetooth system as the number of piconets increases in a given area.

Since Bluetooth operates in the ISM $2.45 \mathrm{GHz}$ band, there are many sources of interference other than Bluetooth devices, e.g. microwave ovens and IEEE 802.11 Wavelans. We, however, focus on the interference generated within the Bluetooth system only ${ }^{~}$. A piconet experiences interference when a frequency hit occurs, i.e., the transmission frequency of a packet in a piconet matches with that being used in one or more other piconets for some overlapping duration. Figure 2 shows ex ample hop sequences for 4 piconets. The first row shows the frequency hit regions of the reference piconet. $T$ denotes the hop time. Note that, the piconets are not synchronised in time. We assume that the time offset of the $i^{\text {th }}$ piconet $(2 \leq i \leq M-1)$, denoted by $t_{i}$, with respect to the reference piconet are uniformly distributed in $[0, T]$.

Since the devices in the piconets are spatially distributed, the interfering piconet is not a fixed location or a device but a random device in that piconet transmitting at a given time. We assume a physically stationary environment (little or infrequent movement) so that the channel is quasi-static and quasiwide-sense stationary. Hence, for the duration of a frequency hit on the single frequency, constant interference power can be assumed to be received from the individual interferers.

We consider a reference piconet and denote by $\{\Gamma(t), t \geq$ $0\}$, the SIR process.

$$
\Gamma(t)=\frac{P_{S}(t)}{\sum_{j=1}^{N(t)} P_{I}^{j}(t)+\frac{N_{0} W}{2}}
$$

where, at time $t, P_{S}(t)$ is the desired signal power, $P_{I}^{j}(t)$ is the interference power due to the $j^{\text {th }}$ interfering piconet and $N(t)$ is the number of interferers. Recall that, in Bluetooth a device can transmit in alternate slots. In addition, a slave can transmit only when polled. Hence, the receiver is not a fixed device and the SIR process we characterise is of the reference piconet as a whole.

\section{A. Outage Probability}

Outage occurs when the bit SIR falls below the resistance ratio (denoted by $\nu$ ) specified by the standard. We denote by

\footnotetext{
${ }^{1}$ IEEE802.11 uses more power than Bluetooth but is a slower hop system ( $50 \mathrm{hops} / \mathrm{s}$ vs $1600 \mathrm{hops} / \mathrm{s}$ in Bluetooth). It remains to be investigated which system will affect the other more. Also microwave ovens collocated with the work space in an office is an unlikely scenario.
} 
$\Gamma_{b}$ the bit SIR and seek the stationary outage probability $P_{o u t}$.

$$
P_{\text {out }}:=P\left(\frac{P_{S}}{\sum_{j=1}^{N_{b}} P_{I}^{j}+\frac{N_{0} W}{2}}<y\right)
$$

$P_{S}$ is the desired signal power and $P_{I}^{j}$ denotes the power received from the $j^{\text {th }}$ interferer. $N_{b}$ denotes the number of interferers in a bit duration. Let $P_{I, n}:=\sum_{j=1}^{n} P_{I}^{j}$. A Bluetooth receiver has $-70 \mathrm{dBm}$ sensitivity level to meet BER $<0.001$. With $1 \mathrm{Mbps}$ bit rate, the noise power is approximately $2.5 \times 10^{-6} \mathrm{~mW}$. Hence ignoring the thermal noise,

$$
\begin{aligned}
P_{\text {out }} & =P\left(P_{S}<\nu \sum_{j=1}^{N_{b}} P_{I}^{j}\right) \\
& =\sum_{n=0}^{M-1} P\left(P_{S}<\nu P_{I, n} \mid N_{b}=n\right) P\left(N_{b}=n\right)
\end{aligned}
$$

Recall that in the indoor environment under consideration, the mean received power is given by the inverse square law and the signal fading is Rician. Thus, given the distance of the reference transmitter $D_{s}=d_{s}$ and $j^{\text {th }}$ interferer $D_{j}=d_{j}$ from the reference receiver, $P_{S}$ and $P_{I}^{j}$ are non-central chisquare distributed with means $\left(d_{s} / d_{0}\right)^{-2},\left(d_{j} / d_{0}\right)^{-2}$ respectively. In the indoor channels $d_{0}=1 \mathrm{~m}$ ([8]). It then follows that $P_{I, n}$ is non-central chi-square distributed with $2 n$ degrees of freedom.

The reference receiver is located randomly in the room. Let $D_{r}$ denote the distance of the receiver from the center of the room and let $F_{D_{r}}($.$) be its cdf. Denote by \underline{D}:=$ $\left(D_{S}, D_{1}, D_{2}, \ldots, D_{n}\right)$ the vector of distances of the $n+1$ transmitters (one reference transmitter and $n$ interferers) from the receiver. Let $F_{\underline{D}}\left(. \mid D_{r}\right)$ denote the conditional distribution of $\underline{D}$ given the location of the receiver. Then,

$$
\begin{aligned}
& P\left(P_{S}<\nu P_{I, n} \mid N_{b}=n\right)= \\
& \int_{0}^{R} P\left(P_{S}<\nu \dot{P}_{I, n} \mid D_{r}=\tilde{r}, N_{b}=n\right) d F_{D_{r}}(\tilde{r}) \\
& P\left(P_{S}<\nu P_{I, n} \mid D_{r}=\tilde{r}, N_{b}=n\right)= \\
& \quad \int_{A} P\left(P_{S}<\nu P_{I, n} \mid \underline{D}=\underline{d}, D_{r}=\tilde{r}, N_{b}=n\right) d F_{\underline{D}}(\underline{d} \mid \tilde{r}) \\
& P\left(P_{S}<\nu P_{I, n} \mid \underline{D}=\underline{d}, D_{r}=\tilde{r}, N_{b}=n\right)= \\
& \quad \int_{0}^{\infty} F_{P_{S}}\left(\nu x \mid d_{s}\right) d F_{P_{I, n}}\left(\dot{x} \mid d_{1}, \ldots, d_{n}\right)
\end{aligned}
$$

where, $A=\left\{\underline{d}: 0<d_{s}, d_{i} \leq R+\tilde{r}, 1 \leq i \leq n\right\}$. Note that, $F_{P_{S}}\left(. \mid d_{s}\right)$ and $F_{P_{I, n}}\left(. \mid d_{1}, \ldots, d_{n}\right)$ are the non-central chi-square distributions of $P_{S}$ and $P_{I, n}$ respectively as discussed above.

\section{B. Distribution of $N_{b}$}

We model the frequency hopping pattern as a discrete time Markov chain; a state denotes a frequency. Hopping patterns of piconets are independent and identically distributed. We consider $\left\{X_{k}, k \geq 0\right\}$ with one-step transition probabilities given as below. This represents the ideal random hopping pattern.

$$
P\left(X_{k+1}=f_{j} \mid X_{k}=f_{k}\right)= \begin{cases}\frac{1}{N_{f}-1} & f_{j} \neq f_{k} \\ 0 & f_{j}=f_{k}\end{cases}
$$

$N_{f}$ is the total number of frequencies available for transmission. Denote by $\tau$ the bit duration and by $K$ the number of bits in a slot(hop); thus $T=K \tau$. It can be shown that the probability of piconet $j$ hitting a bit in the reference piconet is

$$
P_{h i t}=\frac{1}{N_{f}}\left[1+\frac{1}{K}\right]
$$

Independence among the piconets implies that

$$
P\left(N_{b}=n\right)=\left(\begin{array}{c}
M-1 \\
n
\end{array}\right)\left(P_{h i t}\right)^{n}\left(1-P_{h i t}\right)^{M-1-n}
$$

\section{2-State Markov Channel Model}

In order to charactefse the temporal correlation in the outage process, consider a bit $b$ and $N_{b}=n$. If the next bit $b+1$ starts in the same hop frequency as $b$, then $N_{b+1}$ consists of the interferers out of $n$ that remain in the same hop in $b+1$, denoted by $V$, and the new interferers out of $M-1-n$ piconets that were using a different hop in $b$, denoted by $Z$. However, if $b+1$ starts with a new frequency ( $b$ is the last bit in the slot) then $N_{b+1}$ equals the interferers from potential $M-1-V$ piconets. Let this be denoted by $W$. Let $u$ indicate the hop change, i.e., $u=0$ if there is a hop change and 1 otherwise. Then,

$$
N_{b+1}=u(V+Z)+(1-u) W
$$
$V:=\sum_{j=1}^{N_{b}} v_{j}, Z:=\sum_{j=1}^{M-1-N_{b}} z_{j}, W:=\sum_{j=1}^{M-1-V} w_{j}$
where $(B(p)$ denotes the Bernoulli distribution with probability of 1 being $\mathrm{p}), u \sim B(1-1 / K), v_{j} \sim B(1-1 / K)$, $z_{j} \sim B\left(1 / K \times 1 / N_{f}\right)$ and $w_{j} \sim B\left(P_{h i t}\right)$. Recall that, $K$ is the number of bits per slot.

Using Equation 4, we obtain the one step joint distribution and then approximate the outage process by a Markov process. This gives us a 2-state Markov model as follows. We say that the channel is in good state, denoted by ' $g$ ', if $\Gamma_{b} \geq \nu$ or BER on the channel is below the specified value and is in bad state, denoted by 'b', when $\Gamma_{b}<\nu$, i.e., when the outage occurs. The transition probabilities have been derived in Appendix A. Let $P_{i j}$ denote the transition probability of going from state $i$ to state $j$. Then, the outage duration (in number of bits) is geometrically distributed with mean $1 /\left(1-P_{b b}\right)$. Also the outage probability calculated from the Markov model, denoted by $P_{o u t}^{M}$, is given by $P_{g b} /\left(P_{g b}+P_{b g}\right)$.

\section{RESULTS}

We take $R=5 \mathrm{~m}$ and assume that the devices are distributed uniformly in the room with respect to the center of 
the rom. Then if the receiver is at the center, the cdf of $D$, the distance of a random device from the receiver is given by $F_{D}(d)=d^{2} / R^{2}, 0 \leq d \leq R$. Given $D_{r}=\tilde{r}$, the interferers are assumed to be independently and identically distributed in the room. Let $I_{1}(x):=\frac{1}{\pi} \arccos \left(\frac{(\tilde{r}-d+x)^{2}+\tilde{r}^{2}-d^{2}}{2 \tilde{r}(\bar{r}-d+x)}\right)$ and $I_{2}(x):=\frac{1}{\pi} \arccos \left(\frac{(d-\tilde{r}+x)^{2}+\tilde{r}^{2}-d^{2}}{2 \tilde{r}(d-\tilde{r}+x)}\right)$. Then, it can be shown that if $\tilde{r}>R-\tilde{r}$,

$$
\begin{aligned}
& F_{D}(d \mid \tilde{r})= \\
& \qquad \begin{array}{ll}
\int_{0}^{2 d} I_{1}(x) \frac{2(\tilde{r}-d+x)}{R^{2}} d x & 0 \leq d \leq R-\tilde{r} \\
\int_{0}^{R-\tilde{r}+d} I_{1}(x) \frac{2(\tilde{r}-d+x)}{R^{2}} d x & R-\tilde{r}<d \leq \tilde{r} \\
\frac{(d-\tilde{r})^{2}}{R^{2}}+\int_{0}^{R+\tilde{r}-d^{2}} I_{2}(x) \frac{2(d-\tilde{r}+x)}{R^{2}} d x & \tilde{r}<d \leq R+\tilde{r}
\end{array}
\end{aligned}
$$

Similar expressions can be found if $\tilde{r} \leq R-\tilde{r}$. Figure 3 shows the conditional distance distribution $F_{D}(d \mid \tilde{r})$ for $\tilde{r}=$ $0,1,2,3,4,5 \mathrm{~m}$.

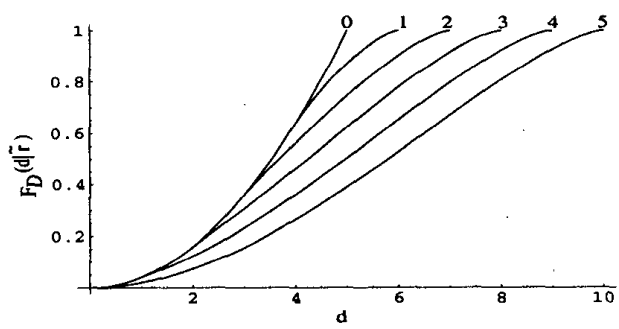

Fig. 3. Distance cdfs for receiver offset $\tilde{r}=(0,1,2,3,4,5) \mathrm{m}$

\section{A. Outage Probability}

Since the calculation of exact outage probability is complicated, even numerically, we resort to finding bounds. Define $g\left(d_{s}, d_{1}, \ldots, d_{n}, \tilde{r}, n\right):=P\left(P_{S}<\nu P_{I, n} \mid \underline{d}, \tilde{r}, n\right)$. Then, Equation 2 can be written as,

$$
\begin{aligned}
& P\left(P_{S}<\nu P_{I, n} \mid D_{r}=\tilde{r}, N_{b}=n\right)= \\
& \quad E_{d_{a} \mid \tilde{r}} E_{d_{n} \mid \tilde{r}} \ldots E_{d_{1} \mid \tilde{r}}\left[g\left(d_{s}, d_{1}, \ldots, d_{n}, \tilde{r}, n\right)\right]
\end{aligned}
$$

Note that $g($.$) is a decreasing function of \left\{d_{i}, 1 \leq i \leq n\right\}$ and an increasing function of $d_{B}$. Also, if $\tilde{r_{1}} \leq \tilde{r_{2}}$ (see Figure 3),

$$
P\left(D_{i}>t \mid \tilde{r_{1}}\right) \leq P\left(D_{i}>t \mid \tilde{r_{2}}\right)
$$

With this stochastic dominance, it follows that, for $1 \leq i \leq n$

$$
E_{d_{i} \mid r_{1}}\left[g\left(\ldots, d_{i}, \ldots\right)\right] \geq E_{d_{i} \mid \tilde{r_{2}}}\left[g\left(\ldots, d_{i}, \ldots\right)\right] \quad \text { if } \tilde{r_{1}} \leq \tilde{r_{2}}(7)
$$$$
E_{d_{s} \mid \tilde{r} 1}\left[g\left(d_{s}, \ldots\right)\right] \leq E_{d_{s} \mid \tilde{r_{2}}}\left[g\left(d_{s}, \ldots\right)\right] \quad \text { if } \tilde{r_{1}} \leq \tilde{r_{2}}(8)
$$

Using Equation 7 and 8 in Equation 5, a lower bound on $P_{\text {out }}$ can be found.

As a simple calculation, assume that there are only two piconets and there is no fading on the channel. Then $P_{S}=$

$$
\begin{aligned}
& \left(\frac{D_{S}}{d_{0}}\right)^{-2} \text { and } P_{I}=\left(\frac{D_{I}}{d_{0}}\right)^{-2} \text {. Then, } \\
& P\left(P_{S}<\nu P_{I} \mid N_{b}=1\right)=P\left(D_{I}^{2}<\nu D_{S}^{2} \mid N_{b}=1\right) \\
& \quad=\int_{0}^{R} P\left(D_{I}<\nu^{1 / 2} D_{S} \mid D_{r}=\tilde{r}, N_{b}=1\right) f_{D_{r}}(\tilde{r}) d \tilde{r} \\
& P\left(D_{I}<\nu^{1 / 2} D_{S} \mid D_{r}=\tilde{r}, N_{b}=1\right) \\
& \quad=\int_{0}^{R+\tilde{r}} P\left(D_{I}<\nu^{1 / 2} d_{s} \mid D_{S}=d_{s}, \tilde{r}, 1\right) d F_{D_{S}}\left(d_{s} \mid \tilde{r}\right) \\
& \quad=E_{D_{s} \mid \tilde{r}}\left[F_{D_{I}}\left(\nu^{1 / 2} d_{s} \mid d_{s}, \tilde{r}\right)\right] \\
& \geq E_{D_{s} \mid \tilde{r}=0}\left[F_{D_{I}}\left(\nu^{1 / 2} d_{s} \mid d_{s}, \tilde{r}\right)\right] \\
& \geq E_{D_{S} \mid \tilde{r}=0}\left[F_{D_{I}}\left(\nu^{1 / 2} d_{s} \mid d_{s}, \tilde{r}=5\right)\right]
\end{aligned}
$$

Note that, Equation 10 follows from Equation 8 and Equation 11 follows from Equation 6 . Approximating $F_{D}(d \mid \tilde{r}=5)$ by a $4^{\text {th }}$ degree polynomial and taking $\nu=25(14 \mathrm{~dB})$, we get $P\left(P_{S}<\nu P_{I} \mid N_{b}=1\right) \geq 0.939992$ and hence with $P\left(N_{b}=\right.$ 1) $=1 / N_{f}, P_{\text {out }} \geq 0.01189$. Table I shows lower bounds on $P_{\text {out }}$ with signal fading.

TABLE I

LOWER BOUNDS ON OUTAGE PROBABILITY FOR $M=2,3$ PICONETS

\begin{tabular}{|l|l|l|}
\hline M-1 & \multicolumn{2}{|l|}{ Lower bound on $P_{\text {out }}$} \\
\hline & $14 \mathrm{~dB}$ & $11 \mathrm{~dB}$ \\
\hline 1 & 0.0114 & 0.0103 \\
2 & 0.0227 & 0.0204 \\
\hline
\end{tabular}

\section{B. Outage Duration}

TABLE II

PARAMETERS OF THE 2-STATE MARKov MODEL

\begin{tabular}{|l|l|l|l|l|l|}
\hline $\mathrm{M}-1$ & $P_{g b}$ & $P_{b g}$ & $P_{o u t}^{M}$ & $E B$ & $E G$ \\
\hline 1 & 0.00005 & 0.005 & 0.010 & 200 & 20000 \\
2 & 0.000055 & 0.0035 & 0.016 & 285 & 18018 \\
\hline
\end{tabular}

Using the derivation of transition probabilities in Appendix $A$, and lower bounds on the outage probability derived in Section V-A we calculate approximate values of the $P_{g b}$ and $P_{b g}$ for $\nu=14 \mathrm{~dB}$ and 400 bits packet size. We also calculate the outage probability from this $\operatorname{Markov} \operatorname{model}\left(P_{o u t}^{M}\right)$, Results are shown in Table II. Table II also shows in bits the mean outage duration (EB) and the mean duration in good state (EG).

\section{Discussion}

It is very complicated to calculate even the approximate values of $P_{\text {out }}$ as the number of piconets increases. We, therefore, have results only for $M \leq 3$ piconets. However, the insight gained with these results allows us to predict the performance for larger values of $M$. The results indicate that when 
a frequency hit occurs it is difficult to maintain the resistance ratio of $14 \mathrm{~dB}$ as specified by the standard. Hence, co-channel interference is avoided mainly through the use of large number of frequencies. For larger values of $M$, we expect that $P_{\text {out }}$ will increase almost linearly, in the form of $\frac{(M-1)}{N_{f}}$. Though the Markov model is only an approximation (as seen from Table II), we expect that when number of piconets is small outages are infrequent (once in 20000 bits) This is because piconets are not time-synchronised and frequency hits occur with probability $\frac{1}{N_{f}}$. However, outages persist for approximately 200 bits which is considerable duration considering $1 / 3$ and $2 / 3$ FEC used in Bluetooth.' Also in certain packet types FEC is not mandatory. As $M$ increases, duration of good state will reduce at the cost of longer outage durations.

\section{CONCLUSIONS}

We considered a typical scenario for Bluetooth piconets and found the outage probability and the mean outage duration of the SIR process. This led us to a 2-state Markov model for the Bluetooth channel. The outage probability appears to increase almost linearly with the number of piconets. Longer outage durations combined with simple FEC schemes imply that to the applications on Bluetooth, the channel will degrade rapidly as the number of piconets increases.

\section{APPENDIX}

\section{DERIVATION OF TRANSITION PROBABILITIES FOR THE} OUTAGE PROCESS

We derive $P_{b b}:=P\left(\Gamma_{b+1}<\nu \mid \Gamma_{b}<\nu\right)$. Derivation of $P_{g g}$ follows similarly.

$$
\begin{array}{r}
P\left(\Gamma_{b+1}<\nu \mid \Gamma_{b}<\nu\right)=\frac{P\left(\Gamma_{b+1}<\nu, \Gamma_{b}<\nu\right)}{P\left(\Gamma_{b}<\nu\right)} \\
P\left(\Gamma_{b+1}<\nu, \Gamma_{b}<\nu\right)=P\left(\Gamma_{b}<\nu\right)-P\left(\Gamma_{b+1}<\nu, \Gamma_{b} \geq \nu\right)
\end{array}
$$

In the following equations, when a random variable is given, it is understood that the value it takes is given by the corresponding small letter.

$$
\begin{aligned}
& P\left(\Gamma_{b+1}<\nu, \Gamma_{b} \geq \nu\right) \\
& \quad=\sum_{n=0}^{M-1} P\left(\Gamma_{b+1}<\nu, \Gamma_{b} \geq \nu \mid N_{b}\right) P\left(N_{b}=n\right) \\
& P\left(\Gamma_{b+1}<\nu, \Gamma_{b} \geq \nu \mid N_{b}\right) \\
& \quad=P\left(\Gamma_{b+1}<\nu, \Gamma_{b} \geq \nu \mid u=1, N_{b}\right) P(u=1) \\
& \quad+P\left(\Gamma_{b+1}<\nu, \Gamma_{b} \geq \nu \mid u=0, N_{b}\right) P(u=0)
\end{aligned}
$$

Note that $u$ is independent of $N_{b}$. Let $P_{I, n}:=\sum_{j=1}^{n} P_{I}^{j}$. From now on we denote $u=1$ by $u_{1}$ and $u=0$ by $u_{0}$. - $u=1 \Rightarrow$ no hop change. Recall that, the channel is assumed to be quasi-static and quasi-stationary. Then constant $P_{S}$ is received for the hop time. Also, for the duration of a hit, constant interference power is received from the individual interferers.

$$
\begin{aligned}
& P\left(\Gamma_{b+1}<\nu, \Gamma_{b} \geq \nu \mid u_{1}, N_{b}\right) \\
& \quad=\sum_{z=1}^{M-1-n} \sum_{v=1}^{n} P\left(P_{S}<\nu P_{I, Z+V}, P_{S} \geq \nu P_{I, n}, Z=z,\right. \\
& V=v \mid u_{1}, N_{b} \downarrow \\
& P\left(P_{S}<\nu P_{I, Z+V}, P_{S} \geq \nu P_{I, n}, Z=z, V=v \mid u_{1}, N_{b}\right) \\
& \quad=P\left(P_{S}<\nu P_{I, z+v}, P_{S} \geq \nu P_{I, n} \mid Z, V, u_{1}, N_{b}\right) \\
& \quad P\left(Z=z, V=v \mid u_{1}, N_{b}\right) \\
& \quad=P\left(\nu P_{I, n} \leq P_{S}<\nu P_{I, z+v} \mid Z, V, u_{1}, N_{b}\right) \\
& \quad P\left(Z=z \mid N_{b}\right) P\left(V=v \mid N_{b}\right)
\end{aligned}
$$

- $u=0 \Rightarrow$ hop changes. Hence, the receiver changes. We indicate by $P_{S}^{b+1}$ and $P_{S}^{b}$ the signal powers received in bits $b+1$ and $b$ respectively. Then,

$$
\begin{aligned}
& P\left(\Gamma_{b+1}<\nu, \Gamma_{b} \geq \nu \mid u_{0}, N_{b}\right) \\
& \quad=\sum_{v=1}^{n} P\left(P_{S}^{b+1}<\nu P_{I, W}, P_{S}^{b} \geq \nu P_{I, n}, V=v \mid u_{0}, N_{b}\right) \\
& P\left(P_{S}^{b+1}<\nu P_{I, W}, P_{S}^{b} \geq \nu P_{I, n}, V=v \mid u_{0}, N_{b}\right) \\
& \quad=P\left(P_{S}^{b+1}<\nu P_{I, W}, P_{S}^{b} \geq \nu P_{I, n}, \mid V, u_{0}, N_{b}\right) \\
& \quad P\left(V=v \mid N_{b}\right) \\
& P\left(P_{S}^{b+1}<\nu P_{I, W}, P_{S}^{b} \geq \nu P_{I, n}, \mid V, u_{0}, N_{b}\right) \\
& \quad=\sum_{w=1}^{M-1-v} P\left(P_{S}^{b+1}<\nu P_{I, W}, P_{S}^{b} \geq \nu P_{I, n},\right. \\
& \left.\quad W=w \mid V, u_{0}, N_{b}\right) \\
& P\left(P_{S}^{b+1}<\nu P_{I, W}, P_{S}^{b} \geq \nu P_{I, n}, W=w \mid V, u_{0}, N_{b}\right) \\
& \quad=P(W=w \mid V) P\left(\nu P_{I, n} \leq P_{S}^{b} \mid W, V, u_{0}, N_{b}\right) \\
& \quad P\left(P_{S}^{b+1}<\nu P_{I, w} \mid W, V, u_{0}, N_{b}\right)
\end{aligned}
$$

since the power distribution at different receivers is independent.

\section{REFERENCES}

[1] H. S. Wang and N. Moayeri, "Finite-State Markov Channel-A Useful Model for Radio Communication Channels," IEEE Transactions on VeModel for Radio Communication Channels,"
hicular Technology, vol. 44, no. 1, pp. 163-17, February 1995.

[2] M. Zorzi, R.R. Rao, and L.B. Milstein, "On the Accuracy of a Firstorder Markov Model for Data Transmission on Fading Channels," in Proc. IEEE ICUPC'95, November 1995, pp. 211-215.

[3] Anurag Kumar, "Comparative Performance Analysis of Versions of TCP in Local Network with a Lossy Link," IEEE/ACM Transactions on Nerworking, vol. 6, no. 4, August 1998.

[4] The Bluetooth Specification, available from htlp://www.bluetooth.com/.

[5] Homayoun Hashemi, "The Indoor Radio Propagation Channel," Proceedings of The IEEE, vol. 81, no. 7, pp. 943-968, July 1993.
.

[6] T. S. Rappaport and C. D. McGillem, "UHF Fading in Factories," IEEE Journal on Selected Areas in Communications, vol. 7, no. 1, pp. 40-48, January 1989.

[7] Robert J.C. Bultitude, "Measurement, Characterisation and Modelling of Indoor 800/900 MHz Radio Channels for Digital Communications," IEEE Communications Magazine, vol. 25, pp. 5-12, June 1987.

[8] Theodore Rappapor, Wireless Communications: Principles and Practice, Prentice Hall, 1996. 